Accretion Phenomena and Related Outflows, IAU Colloquium 163

ASP Conference Series, Vol. 121, 1997

D.T. Wickramasinghe, L. Ferrario, and G.V. Bicknell, eds.

\title{
Magnetospheric Accretion in T Tauri Stars
}

\author{
Philip J. Armitage ${ }^{1}$ \\ Institute of Astronomy, Madingley Road, Cambridge CB3 OHA, UK
}

\begin{abstract}
Many, perhaps most, accreting T Tauri stars show evidence for magnetically dominated accretion flows near the stellar surface. I discuss the implications of this for the rotation rates and long-term photometric variability of $\mathrm{T}$ Tauri stars.
\end{abstract}

\section{Introduction}

Magnetic fields of kilogauss strength have been measured in at least four $\mathrm{T}$ Tauri stars (TTS) via the broadening of Zeeman sensitive spectral lines (Basri, Marcy \& Valenti 1992; Guenther \& Emerson 1996), and indirect evidence suggests that such fields are common in both accreting, Classical T Tauri stars (CTTS), and in Weak-Lined T Tauri stars (WTTS) lacking the signatures of ongoing accretion. As noted by Königl (1991), ordered magnetic fields of this strength are sufficient to disrupt the inner regions of the accretion disk, so that matter flows in along field lines to strike the star as a high latitude accretion column. Observations that support this model of accretion in CTTS include:

- The infrared colours of CTTS disks, which are often redder than predicted if the disk is assumed to extend to the stellar surface (Kenyon, Yi \& Hartmann 1996).

- Spectroscopic evidence for infall at the free-fall velocity in CTTS (e.g. Edwards et al. 1994).

Magnetospheric rather than boundary layer accretion is thus probably a common phenomenon in Classical T Tauri stars (Hartmann 1994).

The ubiquity of a magnetospheric accretion model in TTS has implications for the rotation rates and, more speculatively, the long-term photometric variability of TTS. Here, I present time-dependent models for disks that evolve under the combined influence of internal, viscous torques, and external torques provided by the stellar magnetic field. Time-dependent modelling is necessary when the disk departs from a steady-state, both on long timescales (when the viscous timescale in the outer regions of the disk approaches or exceeds the stellar evolution timescale), and over periods of years if the stellar magnetic field varies on those shorter timescales.

\footnotetext{
${ }^{1}$ Present address: Canadian Institute for Theoretical Astrophysics, University of Toronto, Ontario, M5S 1A7, Canada
} 


\section{Description of the model}

The evolution of the disk surface density $\Sigma(R, t)$ is described by the equation (Livio \& Pringle 1992),

$$
\frac{\partial \Sigma}{\partial t}=\frac{3}{R} \frac{\partial}{\partial R}\left[R^{1 / 2} \frac{\partial}{\partial R}\left(\nu \Sigma R^{1 / 2}\right)\right]+\frac{1}{R} \frac{\partial}{\partial R}\left(\frac{B_{z} B_{\phi} R^{5 / 2}}{\pi \sqrt{G M_{*}}}\right),
$$

where $\nu$ is the kinematic viscosity and $B_{z}, B_{\phi}$ are the components of the stellar magnetic field at radius $R$. We take the stellar field to be a dipole, aligned with the stellar rotation axis and perpendicular to the disk plane. Azimuthal field is generated from $B_{z}$ via shear, while the growth of $B_{\phi}$ may be limited by processes including reconnection in the magnetosphere and turbulent diffusion in the disk. We take for $B_{\phi}(R), B_{\phi}=B_{z}\left[\Omega(R)-\Omega_{*}\right] / \Omega(R)$, where $\Omega(R)$ is the Keplerian angular velocity in the disk, as used by Livio \& Pringle (1992) (see also Wang 1996 , other contributions in this volume). Experimentation suggests that the main effect of adopting alternate prescriptions is to change the values of $B_{*}$ required to match observed stellar rotation periods, while the general features of the evolutionary models remain much the same.

The kinematic viscosity $\nu$ is derived from fits to published vertical structure calculations $\left(\alpha=10^{-3}\right)$, while the stellar model used is a $1 M_{\odot}$ pre-mainsequence model calculated using the Eggelton code (Tout, private communication). The star is assumed to rotate rigidly, and the field scaling with rotation rate is $B_{*} \propto \Omega_{*}$. Full details are given elsewhere (Armitage \& Clarke 1996).

\section{Rotation rates}

Figure 1 shows a typical calculation of the evolution of the stellar rotation period, $P_{*}$. Above some critical disk mass, magnetic braking successfully keeps $P_{*}$ at $\sim 6$ 6-10 days during the CTTS phase, after which the star spins up as a WTTS to $P_{*} \sim 4$ days. The initial disk mass is unimportant provided it exceeds the critical value (here $\sim 10^{-3} M_{\odot}$ ), lower mass disks are rapidly expelled by the magnetic torques and fail to brake the star significantly (c.f. Cameron, Campbell \& Quaintrell 1995). The relative rotation rates of CTTS and WTTS seen in these models are consistent with observations (Bouvier et al. 1995), and the required magnetic fields $\left(B_{*}=500-1000 \mathrm{G}\right.$ for CTTS, $B_{*} \approx 1500 \mathrm{G}$ for WTTS) seem reasonable given the current, limited, measurements and limits.

In this scenario, the lifetime of the disk relative to the timescale on which the star contracts determines the extent to which WTTS rotate faster than CTTS. Close binaries of a few a.u. separation, in which the companion truncates the disk and modifies its lifetime, should therefore influence the rotation rates of WTTS in such binaries. Figure 1 shows an explicit calculation - in this case the inner disk lifetime is prolonged due to the removal of angular momentum at the tidal truncation radius, leading to similar rotation rates whilst the model stars are CTTS but slower spin for the binaries as WTTS.

There are several sources of uncertainty in these models, most seriously the lack of knowledge of when and by what mechanism the disk is cleared at the end of the CTTS phase, but also including the disk viscosity and the MHD problem of 

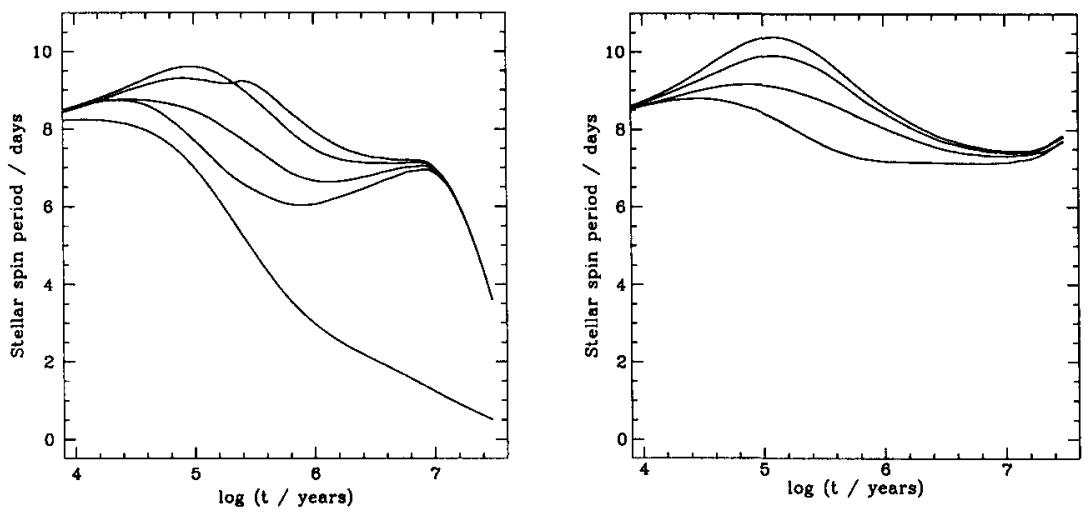

Figure 1. Time dependence of the stellar spin period for models computed with outer boundary conditions appropriate for single (left panel), and binary stars (right panel). From top downwards, the curves at left represent initial disk masses $M_{\text {disk }}=3 \times 10^{-3}, 10^{-2}, 3 \times 10^{-2}$, $10^{-1}$ and $10^{-3} M_{\odot}$ respectively. For the 'binary' runs, the curves show periastron distances of $1,2,4$ and 8 a.u.

determining the twisted field configuration. These results suggest that different rotation rates of close binaries offer one test as to whether magnetic braking via star-disk linkage is responsible for the slow spin of $\mathrm{T}$ Tauri stars.

\section{Variability}

$\mathrm{T}$ Tauri stars are known to be variable in the optical over long timescales of years to decades (Herbst et al. 1994). The temporal behaviour of the magnetic fields in these stars is as yet undetermined from observations. However, dynamo generated fields might plausibly vary over periods of years, and this may contribute to photometric variability of the system via the influence of the stellar field on the disk.

For disks around T Tauri stars in which $\alpha \sim 10^{-3}$, magnetic cycles on observationally interesting timescales lead to relatively small displacements in the magnetospheric radius $R_{\mathrm{m}}$. If $R_{\mathrm{m}}$ remains within the corotation radius $R_{\mathrm{c}}$ throughout the cycle, the resulting accretion rate scales simply as the magnetic torque, $\dot{M} \propto B_{*}^{2}$. However if $R_{\mathrm{m}}$ crosses $R_{\mathrm{c}}$ during the cycle, larger modulation of $\dot{M}$ is produced and accretion occurs in pulses (Figure 2, Armitage 1995). This latter possibility is intriguing as it provides a possible mechanism for converting systems between CTTS and WTTS status (Clarke et al. 1995). In either scenario, order unity variations in $B_{*}$ typically lead to photometric variability of $\sim$ 1 magnitude in $\mathrm{V}$, with the possibility of significant variability (a few tenths of a magnitude) in the infrared up to $10 \mu \mathrm{m}$. Long term observations and historical records might therefore be able to constrain the possibility of variable magnetic fields in T Tauri stars. 

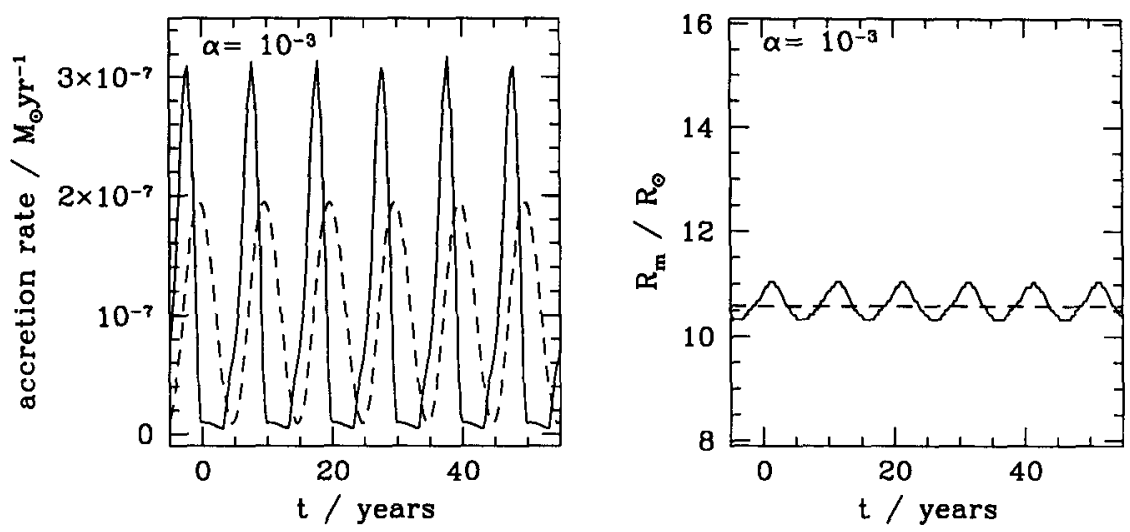

Figure 2. Time-dependence of the accretion rate and magnetospheric radius for a magnetic cycle crossing corotation. The phase of the stellar magnetic field is shown in the left panel as the dashed curve, $R_{\mathrm{c}}$ is shown in the right panel as a horizontal line.

Acknowledgments. Many thanks to Cathie Clarke for her assistance with all aspects of the work presented here.

\section{References}

Armitage, P. J. 1995, MNRAS, 274, 1242

Armitage, P. J., \& Clarke, C. J. 1996, MNRAS, 280, 458

Basri, G., Marcy, G. W., \& Valenti, J.A. 1992, ApJ, 390, 622

Bouvier, J., Covino, E., Kovo, O., Martin, E. L., Matthews, J. M., Terranegra, L., \& Beck, S. C. 1995, A\&A, 299, 89

Cameron, A. C., Campbell, C. G., \& Quaintrell, H. 1995, A\&A, 298, 133

Clarke, C. J., Armitage, P. J., Smith, K. W. \& Pringle, J. E. 1995, MNRAS, 273,639

Edwards, S., Hartigan, P., Ghandour, L., \& Andrulis, C. 1994, AJ, 108, 1056

Guenther, E. W., \& Emerson, J. P. 1996, in The Role of Dust in the Formation of Stars, Siebenmorgen R., Käufl H. U., eds, ESO Proceedings, in press

Hartmann, L. 1994, in Theory of Accretion Disks 2, Duschl W. J. et al., eds, NATO ASI Series C417, Kluwer, p. 19

Herbst, W., Herbst, D. K., Grossman E. J., \& Weinstein D. 1994, AJ, 108, 1906

Kenyon, S. J., Yi, I., \& Hartmann, L. 1996, ApJ, 462, 439

Königl, A. 1991, ApJ, 370, L39

Livio, M., \& Pringle, J. E. 1992, MNRAS, 259, 23P

Wang, Y. -M. 1996, ApJ, 465, L111 


\section{Discussion}

R. Nelson: I would just like to remind everyone again that in the X-Ray pulsars - the only systems where we can actually measure the time dependent accretion torques - the neutron star spin does not reach a nice equilibrium zero torque state, but appears to alternate between episodes of rapid spin-up, followed by a rapid transition to extended spin-down. This behaviour contradicts the standard Ghosh-Lamb theory of magnetosphere-disk interaction.

P. Armitage: I agree with that, though as you also discussed some of those complexities in the X-Ray pulsars may be due to the mass transfer process. 